\title{
Sperm-hybrid micromotors: on-board assistance for nature's bustling swimmers
}

\author{
Lukas Schwarz ${ }^{1}$ Mariana Medina-Sánchez ${ }^{1}$ and Oliver G Schmidt ${ }^{1,2}$ \\ ${ }^{1}$ Institute for Integrative Nanosciences, Leibniz IFW, Dresden, Germany and ${ }^{2}$ Material Systems for Nanoelectronics, \\ Chemnitz University of Technology, Chemnitz, Germany
}

Correspondence should be addressed to M Medina-Sánchez; Email: m.medina.sanchez@ifw-dresden.de

\begin{abstract}
Sperm cells that cannot swim and orient properly compromise male fertility. Such defects are responsible for male infertility regardless of the actual quality of the most important content, the sperm's DNA. Synthetic micromotors are engineered devices that are able to swim in (body) fluids and microscopic environments, similar to flagellated cells like sperm. Coupled together, a spermhybrid micromotor embodies the concept of bringing the sperm cell together with artificial components that assist or replace defective functions of the cell, helping it to pursue its goal without interfering with its health, enabling the process of assisted fertilization and further embryo development all inside the body. Non-invasive, remote-controlled in vivo applicability is the key quality of such hybrid microdevices. Assisted reproduction with the help of micromotors is in the focus of this review, although other biomedical applications that arise from the powerful combination of sperm cell and synthetic enhancement are also discussed and summarized. Details are provided about different fabrication processes and cell-material coupling strategies, and the way from proof-of-concept studies to in vivo experiments in animals is outlined.

Reproduction (2020) 159 R83-R96
\end{abstract}

\section{Introduction}

Sperm cells are intriguing lifeforms, from the theoretical question of whether they can be qualified as such to the practical question of whether a male can successfully reproduce with his or not. The two main qualities of a sperm cell that enable it to continue the life cycle of its species - at least in the case of animal spermatozoa - are the haploid DNA that it carries and its motility. Both qualities have been investigated extensively by biologists and physicians since their discovery, leading to successful developments in assisted reproduction, breeding, and birth control. At present, physicists and engineers also enter this field to contribute with their expertise. From this perspective, sperm's motility is the first point of attraction as it is one of nature's most impressive micromechanical technologies. Already serving as an inspiration for decades to create motion at the microscale (Purcell 1977), sperm cells have recently been in focus to be modified and applied themselves as microscale actuators with synthetic enhancements (Magdanz et al. 2017, Medina-Sánchez et al. 2017). In this review, such sperm-hybrid microdevices are discussed, which highlight unconventional approaches to assist sperm cells in their task of fertilization and serve to facilitate assessing and investigating sperm behavior or employing sperm cells as motor units for other applications in the body, most prominently targeted drug and gene delivery. First, we briefly review the sperm cell's general properties - especially regarding motility and taxis - and place them in relation with state-of-theart synthetic micromotors. Subsequently, different ways of combining sperm with microscopic attachments to a sperm-hybrid micromotor are discussed. Finally, applications of such hybrid devices are presented in a comprising review of the corresponding literature, exploring the challenges and opportunities of spermhybrid micromotors in assisted reproduction, as well as targeted drug delivery.

\section{Fundamentals}

\section{Sperm morphology and locomotion}

Essentially, the egg cell is responsible for providing nutrients for the development of a potential embryo, whereas the sperm cell is responsible for bringing both haploid genomes together with its motility to enable fusion. Furthermore, both germ cells also engage in competition and selection procedures and interact with the environment in the female reproductive tract, which requires the sperm cell to be either large and durable or numerous (Snook 2005, Bianchi et al. 2014). Larger mammals usually employ the latter strategy, although this does not make their individual sperm cells' abilities any less impressive. 
First of all, despite different habitats and reproduction strategies that lead to numerous sperm shapes and sizes in the animal kingdom, the basic structure of the head, midpiece, and tail remains the same for most species (Alvarez 2017). Especially the tail - which is responsible for motility - is based on a structure that is surprisingly consistent in nature, namely the axoneme comprising strands of microtubules with dynein motor proteins that are arranged in pairs in the so-called ' $9+2$ ' configuration (Fig. 1). This well-investigated architecture is explained and illustrated in detail (for example, in Gaffney et al. 2011). Unlike bacterial flagella, which are actuated at the connecting hook between the head and tail, the whiplash beating pattern of eukaryotic flagella like the sperm tail emerges from concerted action of motor proteins all along the axoneme. The resulting beating frequency, amplitude, and swimming velocity significantly vary between species and to some extent within one semen sample.

The ability to navigate through the female reproductive tract - often a complex, obstructed and even hostile environment - is the key property of mammalian sperm to successfully deliver the haploid nucleus in their head to the egg cell. Whereas the actual fusion of sperm and oocyte is mainly a biochemical process triggered by the so-called acrosome reaction of the sperm head, the preceding DNA transport - that is, the swimming journey of the sperm cell - is a mechanical process that is therefore in the focus of our interest as physicists and engineers, not only to provide theoretical groundwork and practical calculations to understand the motion patterns of sperm like in several insightful works (Lauga and Powers 2009, Saggiorato et al. 2017, Kromer et al. 2018, Rode et al. 2018) or to reconstruct their journey through the reproductive tract, but also to technically support or even exploit their swimming capability as natural micromotors.

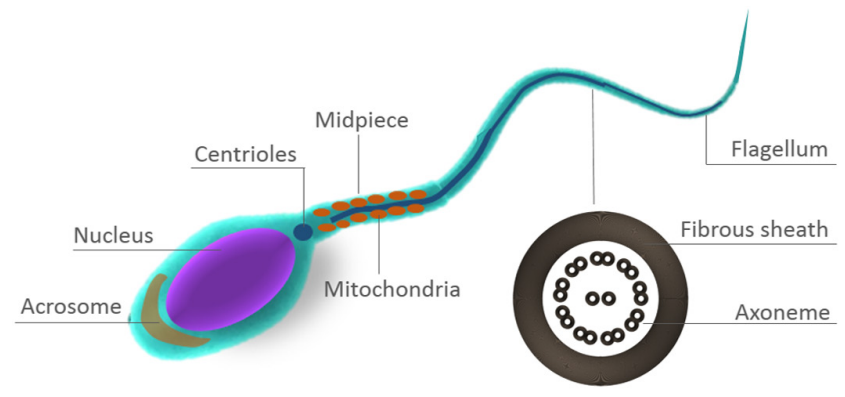

Figure 1 General sperm cell morphology. The sperm head contains the haploid nucleus. During the acrosome reaction, the cell membrane of the head's front part will open up to release chemicals and bond to the egg cell's zona pellucida. The midpiece contains mitochondria to supply chemical energy for the cell. The flagellum is comprised of the fibrous sheath that contains centrioles that make up the axoneme with $9+2$ pairs of microtubules with dynein motor proteins that are responsible for the whiplash stroke of the tail by sliding against each other.
The dimensions and swimming velocities of some sperm species that are commonly investigated as model systems or for potential micromotor applications are summarized in (Fig. 2). Bovine (bull) spermatozoa and those of other large mammals are similar in shape to human sperm cells, albeit with varying head and tail sizes (Cummins and Woodall 1985). Those of smaller mammals - especially murine (mouse) spermatozoa, which are abundant in research - are generally larger with much longer tails and feature a hook-like structure at the head (Chang et al. 2013). Due to their size, they are also faster swimmers, at least considering absolute velocity in similar cell culture medium (Chang et al. 2013). The sperm cells of maritime, invertebrate species like sea urchins and sea squirts (ascidians) have become well-established systems for fundamental investigations due to their good availability, although they are also interesting candidates for sperm-hybrid micromotors given their swimming performance and chemotaxis capabilities, which are adapted to swimming in the open sea (Chang et al. 2013, Chen et al. 2018).

Considering assisted reproductive technology (ART), of all the sperm-related diagnoses for male infertility - aspermia (no semen), hypospermia (small semen volume), azoospermia (no sperm cells), teratozoospermia (abnormal sperm morphology), necrozoospermia (dead sperm cells), asthenozoospermia (low sperm motility), and oligozoospermia (few sperm cells) - the latter two seem susceptible to being remedied by mechanical engineering of the individual sperm cell. In the case of asthenozoospermia by technically improving or restoring sperm motility, in the case of oligozoospermia in a similar way by providing the individual sperm cell with means to successfully make its way to the oocyte, that is, to circumvent the disadvantage of low numbers by strengthening the individual capability to succeed. The key advantage of such 'on-board' support approaches - in comparison with conventional in vitro fertilization (IVF) and intracytoplasmic sperm injection (ICSI) - is the potential in vivo applicability, that is, the possibility to fertilize with microrobot-assisted sperm cells in the natural environment of the female reproductive tract and therefore avoid explantation of the oocyte to the laboratory. In this sense, microrobot-assisted ART can be understood as an extension of intrauterine insemination (IUI) with means of the 21 st century to treat cases of male infertility where otherwise ICSI would be unavoidable. However, when applied in an in vitro setup, sperm-hybrid micromotors may have advantages over ICSI, considering the invasiveness of the latter technique. In this context, not only is motility important but also the sperm cell's navigation abilities, that is their taxes responses.

\section{Sperm taxis and cooperative action}

Although several factors have been identified that potentially influence the sperm cells' journey through 


\begin{tabular}{|c|c|c|c|c|c|c|}
\hline Shape & Origin & Head length & Head width & Tail length & Velocity & References \\
\hline & Bovine & са. $10 \mu \mathrm{m}$ & ca. $5 \mu \mathrm{m}$ & ca. $60 \mu \mathrm{m}$ & Up to $100 \mu \mathrm{m} / \mathrm{s}$ & $\begin{array}{l}\text { Cummins \& Woodall 1985, Magdanz } \\
\text { et al. } 2013\end{array}$ \\
\hline & Human & ca. $5 \mu \mathrm{m}$ & ca. $3 \mu \mathrm{m}$ & ca. $60 \mu \mathrm{m}$ & Up to $70 \mu \mathrm{m} / \mathrm{s}$ & $\begin{array}{l}\text { Harvey 1960, Cummins \& Woodall } \\
\text { 1985, Geerts et al. } 2014\end{array}$ \\
\hline & Murine & ca. $8 \mu \mathrm{m}$ & ca. $4 \mu \mathrm{m}$ & ca. $100 \mu \mathrm{m}$ & Up to $300 \mu \mathrm{m} / \mathrm{s}$ & $\begin{array}{l}\text { Cummins \& Woodall 1985, Chang et al. } \\
\text { 2013, Geerts et al. } 2014\end{array}$ \\
\hline & Ascidian & ca. $4 \mu \mathrm{m}$ & са. $1 \mu \mathrm{m}$ & ca. $50 \mu \mathrm{m}$ & Up to $250 \mu \mathrm{m} / \mathrm{s}$ & Chen et al. 2018 \\
\hline
\end{tabular}

Figure 2 Sperm dimensions and swimming velocity: Comparison of different sperm species.

the female reproductive tract, their exact interplay and relative importance remains elusive (Kaupp and Strünker 2017). The female reproductive tract provides a variety of physical and chemical clues that impede or stimulate the traveling sperm cells, which have a set of abilities to react accordingly (Suarez and Pacey 2006). A first means to orientate themselves in the comparatively vast environment inside the uterus - after passing the hostile, low-pH vagina and cervix region by ejaculation and sheer numbers - is their tendency to swim preferably along walls, that is, endothelium (Fauci and McDonald 1995, Denissenko et al. 2012, Rode et al. 2018). This behavior can be considered as a form of thigmotaxis, that is, the ability to follow a touch stimulus (Magdanz et al. 2015a). Inside the uterus, peristaltic pumping by muscle contractions of the epithelial surroundings seems to be the major factor to transport sperm, as it has been reported that sperm cells can be found inside the fallopian tubes only seconds after ejaculation, which would otherwise not be possible simply by flagellar swimming with a speed of several tens of micrometers per second (Brannigan and Lipshultz 2008).

After entering the fallopian tubes, peristaltic action might still play a role, although here sperm are actually aligning and swimming against a flow of liquid. This behavior is called rheotaxis (Miki and Clapham 2013). In the confined environment of the oviduct, other forms of taxes also come into play that have not been consistently evaluated yet in this context, namely chemotaxis and thermotaxis, that is, the ability to pick up and follow a chemical gradient and a temperature gradient, respectively. A slight temperature gradient toward the oocyte in the fallopian tube has been reported in mammals (Bahat and Eisenbach 2006). Moreover, the oocyte is known to secrete several chemical cues that attract sperm cells in various species (Friedrich and Jülicher 2007, Kaupp and Strünker 2017). However, in human medicine it remains debated whether these factors are sufficient or even required for successful fertilization in vivo. The same applies for the adherence of sperm cells to oviduct epithelium cells, which seems to serve sperm storage but may also induce sperm capacitation (Coy et al. 2012, Suarez 2016).

Sperm capacitation and hyperactivation are important alterations of individual sperm performance that are triggered chemically by the surroundings in the oviduct (Brannigan and Lipshultz 2008). Most importantly, the beating frequency and amplitude of the sperm flagellum significantly increase. This is apparently necessary to penetrate the cumulus oophorus complex around the oocyte, a procedure that also illustrates another important factor, namely the sperm cells' influence on each other. There are studies on how sperm of the wood mouse band together to move and overcome obstacles more efficiently (Moore et al. 2002). Furthermore, hydrodynamical calculations serve to illustrate how sperm tail-beating patterns may influence each other and synchronize (Yang et al. 2008). Another interesting mechanism is the release of hyaluronidase after the acrosome reaction, a biochemical process that follows capacitation, which is necessary to trigger the fusion of sperm and egg cell once the sperm cell has adhered to the oocyte's zona pellucida, although it may also happen prematurely (Moore et al. 2002, Brannigan and Lipshultz 2008). Prematurely released hyaluronidase from multiple sperm cells serves to cleave the hyaluronan connections between cumulus oophorus cells and thus facilitates the penetration of sperm in general.

Synthetic components of hybrid micromotors for assisted reproduction may not only serve to restore sperm motility but also support or replace one or more of the capabilities mentioned in this paragraph. Furthermore, microstructures directly attached to sperm cells may also serve as sensors to assess and understand such advanced sperm functions and behavior. The following paragraph summarizes what synthetic microdevices can do and how they might complement sperm cells in a hybrid micromotor approach. 


\section{Synthetic micromotors - how they work and what they can do}

Box 1 provides a brief summary of what micromotors are. Their two basic qualities are motility and a certain level of either controllability or autonomy, or both. There are various kinds of actuation mechanisms and they are generally categorized according to their propulsion by either chemical, physical, or biological means, that is, by internal catalytic reactions, externally applied force fields, or flagellated cells (Medina-Sánchez and Schmidt 2017).

The main difficulties with propulsion at the microscale are the relatively large effect of viscous drag - which demands unconventional swimming strategies (Purcell 1977) - and the small size of microswimmers, which makes the design and fabrication of efficient on-board engines with fuel or power sources very ambitious. For example, inspired by nature's microswimmers, rotating bacterial flagella (Ghosh and Fischer 2009, Zhang et al. 2009, Medina-Sánchez et al. 2016) and beating eukaryotic flagella (Dreyfus et al. 2005, Khalil et al. 2014) were reproduced synthetically, albeit without on-board molecular motor units and instead relying on the energy of externally applied magnetic fields to actuate the microstructures. Moreover, so-called bubblejet micromotors were conceived, tubular microstructures propelled by a chemical reaction that creates gas bubbles that are ejected from one side of the motor and thus push it forward (Mei et al. 2008, Solovev et al. 2009, MedinaSánchez and Schmidt 2017). These microswimmers depend on suitable fuel that needs to be present in the surroundings, which has been shown - for example - with gastric acid in the mouse stomach for in vivo applications (Gao et al. 2015). Finally, instead of the biomimetic imitation of natural microswimmers, the cellular organisms themselves can be employed in a biohybrid approach to transport synthetic loads; for example, therapeutics (Schwarz et al. 2017, Bente et al. 2018).

Depending on the intended application, different designs and strategies may be chosen to achieve the necessary requirements. For example, for the prominent application of targeted drug delivery for tumor treatment, drug encapsulation and controlled release, and simultaneously guiding a multitude of carriers is more important than individual swimming speed and perfect long-term biocompatibility. For the application of assisted fertilization, sperm-hybrid microdevices should leave the sperm cell as intact and unaffected as possible and only take advantage of synthetic components to restore and assist defective or compromised sperm behavior where necessary. Table 1 summarizes the key properties of sperm cells and ways to reproduce or assist them synthetically.

\section{Integration of sperm-hybrid systems}

Conventionally, sperm cells are handled manually with pipettes. In recent studies, the manipulation of sperm cells by liquid flows in microfluidic channels - often as part of so-called lab-on-a-chip systems - has been demonstrated (Knowlton et al. 2015, Nosrati et al. 2017), as well as the capture and fixation of individual sperm cells by optical tweezers (Ohta et al. 2010). However, sperm-hybrid micromotors rely on the direct contact between cell and synthetic component on the microscale, allowing remote control of the individual sperm cell and direct sensing of its behavior in response to external stimuli that may be channeled through the synthetic attachment. This paragraph explains how such microscale attachments are fabricated and how sperm cell and synthetic component are integrated, that is, brought together.

In order to investigate sperm cell behavior, one important aspect is to sort and isolate them and possibly fixate individual ones. As mentioned above, lab-ona-chip systems have been conceived that employ microfluidic flows to guide and manipulate sperm cells, and hydrodynamic trapping to fix them (Knowlton et al. 2015, Nosrati et al. 2017). Moreover, optical tweezers have been applied (Ohta et al. 2010). Synthetic microdevices that can be attached to sperm cells to create sperm-hybrid micromotors offer the advantage of having an on-board sensor system whose interference with the cell's natural behavior can be tailored on-demand.

An easy way to capture an individual sperm cell is to let it swim into a dead end. By means of microfabrication, microscale dead end structures like cones can be created that fit around the head of a single sperm cell. For example, slightly conical microtubes have been fabricated by conventional lithography followed by strain-engineered roll-up of thin layers (Magdanz et al. 2013). These structures attach to sperm

Box 1: Synthetic and hybrid micromotors for biomedical applications.

Micromotors are devices with dimensions at the microscale $(<1000 \mu \mathrm{m})$ that can convert energy to propel themselves, usually to swim in a liquid. Ideally, they do so in an externally controllable manner and are able to carry out tasks like the transport of cargo. Sperm cells are natural micromotors, although engineered micromotors are often purely synthetic and driven - for example - by chemical reactions, light, or magnetic fields. In a hybrid approach, natural micromotors like sperm cells or motile bacteria like E. coli can also be used to propel synthetic attachments and cargo in a controllable way. Micromotors are often developed for potential in vivo applications, that is, biomedical applications in the body, like targeted drug delivery, microsurgery, or diagnostic biosensing. The topic of this review - spermhybrid micromotors - are amalgamations of sperm cells and synthetic components that serve to investigate, enhance, or take advantage of sperm behavior to pursue assisted reproduction or other applications like targeted drug or gene delivery. 
Table 1 General sperm properties: Overview and comparison of physically relevant properties of sperm cells and how they can be complemented synthetically.

\begin{tabular}{|c|c|c|c|c|}
\hline Ability & Sperm cell & Comment & Synthetic enhancement & Comment \\
\hline Swimming & $\begin{array}{l}\text { Single eukaryotic } \\
\text { flagellum }\end{array}$ & Velocity ca. $50 \mu \mathrm{m} / \mathrm{s}$ & $\begin{array}{l}\text { Mostly catalytic engines, magnetic } \\
\text { propellers, ultrasound resonators }\end{array}$ & $\begin{array}{l}\text { ca. } 1-1000 \mu \mathrm{m} / \mathrm{s} \\
\text { (usually at low end) }\end{array}$ \\
\hline Taxis & $\begin{array}{l}\text { Chemo-, thigmo-, } \\
\text { thermo-, rheotaxis }\end{array}$ & $\begin{array}{l}\text { Not clearly } \\
\text { understood }\end{array}$ & Mostly chemo-, magneto-, phototaxis & Visual feedback required \\
\hline Cargo transport & $\begin{array}{l}\text { Protected by cell } \\
\text { membrane }\end{array}$ & Capacity ca. $20 \mu \mathrm{m}^{3}$ & $\begin{array}{l}\text { Nanoparticles, adsorbed layers, } \\
\text { hydrogels, liposomes }\end{array}$ & $\begin{array}{l}\text { Higher loads per unit } \\
\text { possible }\end{array}$ \\
\hline Triggered cargo release & $\begin{array}{l}\text { Acrosome reaction, } \\
\text { cell penetration }\end{array}$ & Biochemical reaction & $\begin{array}{l}\text { Mechanical pick-and-place or shape } \\
\text { changes by external stimuli }\end{array}$ & $\begin{array}{l}\text { Magnetically, by } \mathrm{pH}, \\
\text { light, or temperature }\end{array}$ \\
\hline
\end{tabular}

cells passively when one is swimming into it at random, which happens reliably at a suitable concentration of cells and microstructures (Fig. 3A).

Sperm cells can also be captured actively and selectively by synthetic micromotors. Magnetic microhelices fabricated by 3D laser lithography have been shown to present a suitable geometry to achieve this (Medina-Sánchez et al. 2016). With a sufficiently large diameter to enclose the sperm tail along their axis inside their lumen, but smaller than the diameter of the sperm head, such helices have proven able to wrap around the sperm tail and push the sperm forward at its neck when actuated to rotate in an externally applied magnetic field (Fig. 3B). Such a structure does not rely on the sperm cell's actions but it imposes its own motility onto the captured cell. Therefore, it is necessary to immobilize the target sperm beforehand or specifically target immotile sperm cells, which was indeed the intended purpose in the cited work.

Both kinds of synthetic attachments to sperm - passive shell structures or actively attached motor units - should offer some means to release the captured sperm cell, depending on the intended application. In the case of the magnetically propelled microhelix, the confined sperm cell can be simply released by reverting the direction of rotation of the externally-applied magnetic field to let the helix swim backward away from the sperm head, and set the enclosed sperm tail free again. Synthetic parts that are not motile on their own - like the aforementioned conical tubes - require a different approach. It has been demonstrated that microtubes fabricated by strain-engineered roll-up can also be made from thermoresponsive polymer material, for example, poly $(\mathrm{N}$-isopropylacrylamide), which enables the tube to unroll upon a slight temperature increase and release the confined sperm cell in the process (Magdanz et al. 2016) (Fig. 3C). Such microstructures that can respond to external stimuli and change their behavior as a result of their inherent material properties have recently attracted increasing attention in microengineering and are termed soft and smart materials (Medina-Sánchez et al. 2018). A spring-like mechanism that can bend elastically triggered by hitting a wall or a target object - can be considered as a simplified version of such a stimuliresponsive soft material (Xu et al. 2018) (Fig. 3D).
Finally, a different strategy to functionalize a sperm cell with synthetic components is to let nanoparticles or other entities that are significantly smaller than the sperm attach to its membrane or even enter its cytosol (Fig. 3E). The former can be achieved - for example - by physisorption via Van-der-Waals forces or by covalent bonding via antibody functionalization, whereby the latter is possible with molecules or particles that are sufficiently small to penetrate the sperm membrane, usually in a range below a $50 \mathrm{~nm}$ diameter. These cases offer the advantage of the simple and less time-consuming integration of various synthetic materials, although they suffer from the disadvantage of substantially interfering with the sperm cell's organism, which makes them unfit for fertilization in most cases. However, for applications other than fertilization, these hybrid micromotors present an interesting approach.

In order to increase efficiency, assisted reproduction as well as other applications of sperm-hybrid micromotors will usually require the control and manipulation of multiple sperm cells at the same time. An external magnetic field offers the advantage of being able to guide multiple individual sperm-hybrid devices with one single stimulus. Another strategy is to bind multiple sperm cells to only one synthetic entity

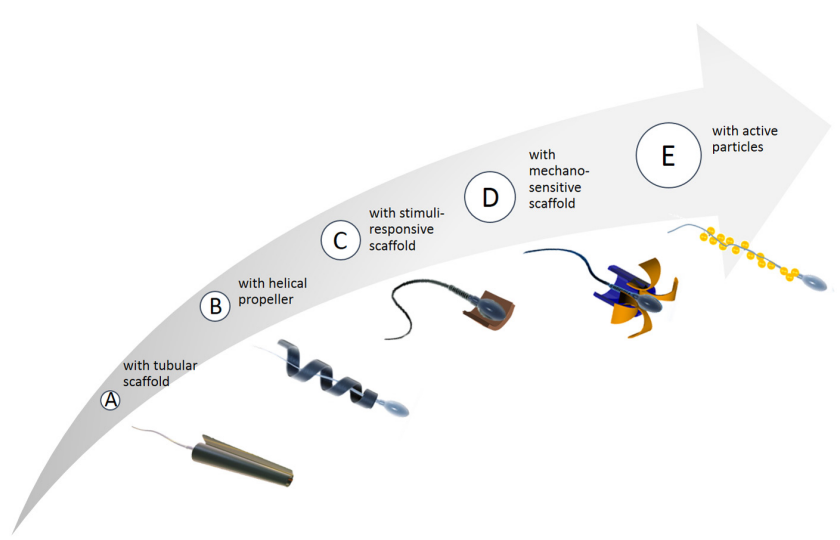

Figure 3 Types of sperm-hybrid systems; sperm cell with (A) slightly conical microtube scaffold, (B) microhelix propeller, (C) stimuliresponsive scaffold, (D) Tetrapod scaffold with flexible arms, and (E) functionalized micro- and nanoparticles; see also (Magdanz et al. 2017, Medina-Sánchez et al. 2017). 
that can be controlled externally. In principle, this is possible for the aforementioned approach to let sperm swim into a conical structure. Moreover, sperm-binding functionalization with chemicals like hyaluronan might be employed to flock sperm cells together to a bundle with aligned sperm tails. Depending on the intended application, necessary sperm release might become more challenging in these cases. The main applications of sperm-hybrid micromotors - along with the accompanying difficulties - are discussed in detail in the following section with reference to corresponding proof-of-concept studies.

\section{Application of sperm-hybrid systems}

\section{Assisted reproduction}

The principal motivation to work with sperm cells is to diagnose and treat male infertility, that is, for assisted reproduction. The first idea and work that presented a sperm-hybrid system as a 'micro-bio-robot' for fertilization were filed (Magdanz et al. 2012) and published (Magdanz et al. 2013) in 2012 and 2013, respectively. In this concept, individual motile sperm cells are confined inside slightly conical microtubes, as described in 'Integration of sperm-hybrid systems' section. It was shown for the first time how sperm cells can be guided due to a synthetic attachment that responds ferromagnetically to an externally applied field (Fig. 4A). The medical benefit lies in the possibility to actively guide swimming sperm to the oocyte in vivo and thus facilitate their journey, especially if they have swimming speed or orientation deficiencies or if there are few, that is, in the case of oligozoospermia. Furthermore, this work also exemplified the idea of having a motile sperm cell actively carry a synthetic attachment. This was an important step to explore the employment of sperm cells for applications other than fertilization, most importantly for targeted drug delivery, which will be discussed later.

The follow-up study on this work showed how such sperm-hybrid micromotors can be tuned by chemical functionalization of the synthetic component (Magdanz et al. 2015b). In this work, two aspects were targeted. By functionalizing the inner surface of the tubes with fibronectin, the coupling efficiency between tube and sperm cell could be improved, that is the probability of a sperm cell being captured inside a conical microtube could be increased by altering the surface properties of the synthetic components to suit the cell's biochemical preferences. Furthermore, means to externally influence the swimming speed of the sperm-hybrid system were investigated by adding caffeine, which proved to temporarily increase the beating frequency of the sperm tail (Fig. 4B). Naturally, the tube dimensions also influence both coupling efficiency and swimming speed, which was exemplified with tubes of 20 and $50 \mu \mathrm{m}$ length (Fig. 4B).
Thefundamental issueof releasing thesperm cell from the microtube after successful delivery was tackled in another study (Magdanz et al. 2016). The synthetic microtubes were fabricated from poly( $\mathrm{N}$-isopropylacrylamide) (PNIPAM), a polymer that has thermoresponsive swelling properties. The material's composition was engineered to exhibit spontaneous swelling in aqueous solutions upon heating over a threshold temperature of $38^{\circ} \mathrm{C}$ (Fig. 4C). Induced by moderate, localized heating, a sperm-hybrid microswimmer swells in physiological medium, which causes the synthetic microtube to unroll and release the confined sperm cell. This was shown in a proof-of-concept experiment. In vivo, localized heating could be achieved either by incorporating magnetic nanoparticles into the tubes, which could be heated by carefully executed magnetic hyperthermia - a technique that is also used to locally heat and kill cancer cells (Giustini et al. 2010) - or the microtube material might be fine-tuned to respond to the slight temperature increase in vicinity to the oocyte, an effect that was also indicated to be perceived by sperm cells that consequently showed thermotaxis toward the oocyte in mammals (Bahat and Eisenbach 2006).

In order to treat a more severe case of male infertility - complete asthenozoospermia, that is, when sperm are unable to swim at all - externally actuated synthetic microswimmers were designed to capture and propel individual sperm cells (Schmidt 2014, MedinaSánchez et al. 2016). Similar to the aforementioned tubular devices, the advantage of this sperm-hybrid approach lies in its potential in vivo applicability, which would allow fertilizing the oocyte directly inside the oviduct by sperm cells with individual microrobotic assistance. Like in the mentioned study, magnetic fields are a powerful tool to control such medical microdevices externally. Untethered, remote control and manipulation of a free-swimming micromotor is thereby possible in a biocompatible, medical-approved setup. In order to minimize the necessary field intensity, no magnetic pulling or pushing with strong gradient fields is employed, but rather magnetic reorientation and alignment to constantly rotating fields. In the discussed study, ferromagnetic microhelices follow the rotation of such an external magnetic field and propel in a corkscrew motion due to the drag anisotropy of the microstructures in a liquid medium (Fig. 4D). This motion principle is well known and outlined in various studies, as it is also the basic swimming strategy of motile bacteria like E. coli, albeit not actuated magnetically but with an on-board molecular motor in this case (Purcell 1977). The coupling of microhelices to individual sperm cells has already been discussed in 'Integration of sperm-hybrid systems' section. It was shown that a metal-coated polymer helix can be actuated externally by a rotating magnetic field to wrap around the tail of an immotile sperm cell and push it at its neck to propel forward. The captured sperm cell was delivered to a 
A
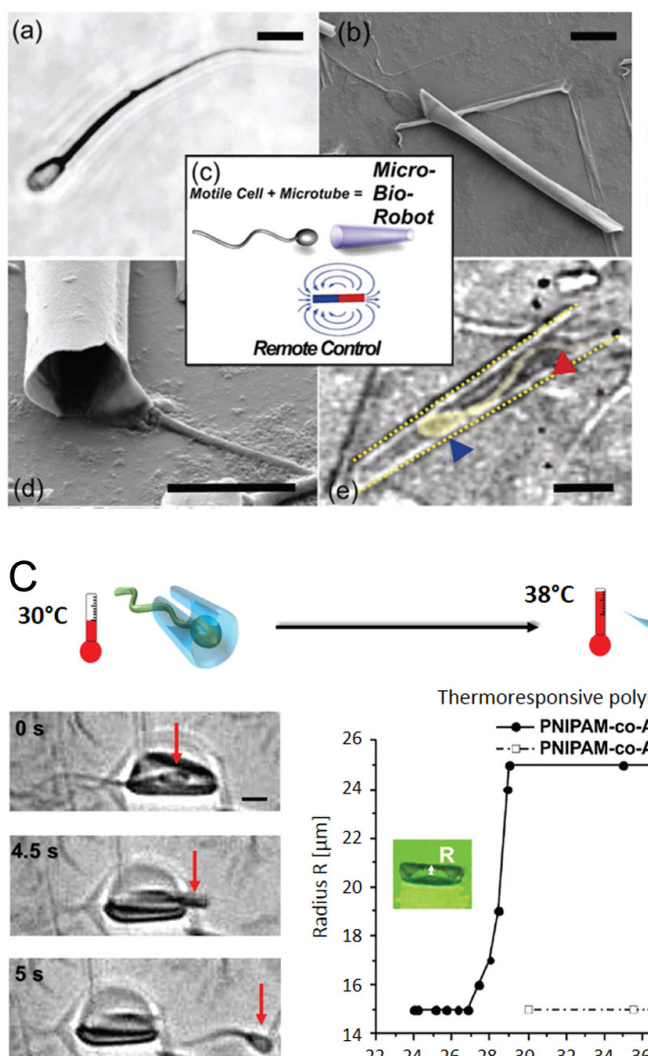

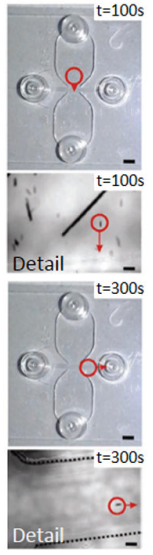

B
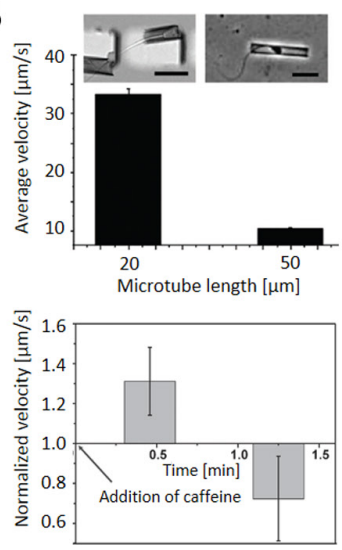

$\mathrm{D}$
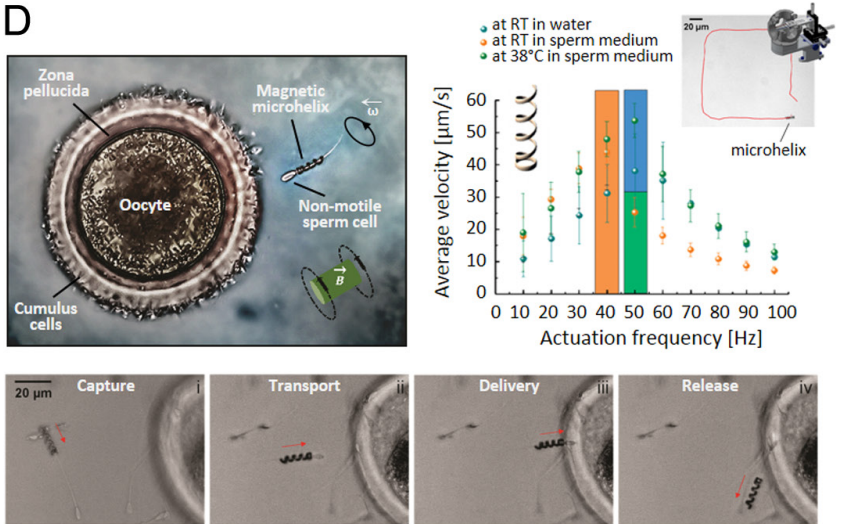
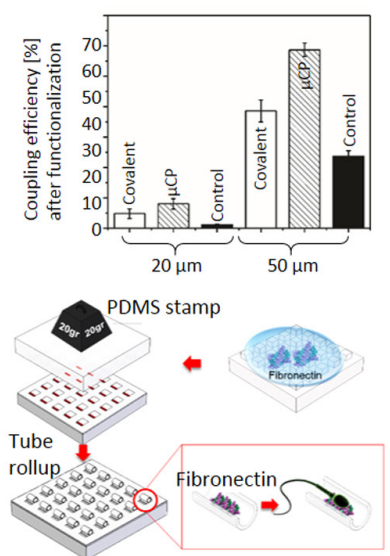

Actuation frequency $[\mathrm{Hz}]$

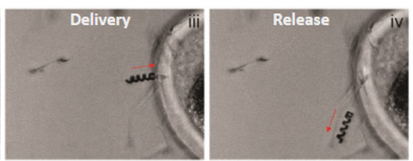

Figure 4 Sperm-hybrid micromotors for assisted fertilization. (A) Motile bovine sperm cell coupled to magnetic metal microtube for magnetic guidance (left side, panels (a)-(e)), live observation and steering in microfluidic channel (right side) (Magdanz et al. 2013). (B) Study of swimming velocity of tubular sperm-hybrid microbots depending on tube length (left side, top graph) and caffeine addition (left side, bottom graph), functionalization of microtubes with fibronectin (right side, bottom scheme) and the influence of functionalization and tube length on coupling efficiency (right side, top graph) (Magdanz et al. 2015b). (C) Temperature-triggered release of confined sperm cells in thermoresponsive PNIPAM microtubes: scheme (top), real-time microscopy video still frames (bottom left), and temperature-tube shape (radius)-dependence (bottom right) (Magdanz et al. 2016). (D) Delivery of immotile bovine sperm cell to the oocyte with magnetic microhelix: scheme and real-time microscopy video still frames (top left and bottom, respectively, see also Video 1), swimming performance of Nickel/Titanium-coated polymer microhelices: field rotation frequency-swimming velocity-dependence in water and sperm culture medium at room temperature and $38^{\circ} \mathrm{C}$ (top right, smaller image: video track of swimming helix actuated by external rotating magnetic field) (Medina-Sánchez et al. 2016).

denuded oocyte and released at the zona pellucida by reversing the rotation of the helix (Fig. 4D and Video 1).

\section{Video 1}

Immotile sperm transport by an helical micromotor. The video (http:// movie-usa.glencoesoftware.com/video/ 10.1530/REP-19-0096/video-1) from theonlineversion of the article is available at https://doi.org/10.1530/ REP-19-0096.

The presented works describe novel strategies to treat male factor infertility - namely oligozoospermia and asthenozoospermia - by providing microrobotic assistance to individual sperm cells to compensate their deficiencies. The approaches were validated in proof-ofconcept studies with bovine sperm and oocytes in in vitro setups with common gamete cell culture media. There are three major challenges to further these efforts. First, the efficiency of sperm delivery needs to be improved, that is, in order to increase fertilization probability, multiple sperm-hybrid devices need to be controlled at the same time, or one synthetic microdevice should support a multitude of sperm cells. Second, it is necessary to establish a suitable imaging technique that allows the visualization of sperm-hybrid devices in vivo. Third, experiments need to be conducted in physiological conditions - that is, in vitro with explanted tissue and body fluids, or in vivo with animal studies - to test the microrobotic strategies under realistic conditions. These challenges are very similar for other biomedical micromotors for in vivo applications, and thus, they will be discussed at the end of the following section. 


\section{Targeted drug therapy}

Biomedical microswimmers for in vivo applications have been widely investigated for targeted drug delivery to treat various diseases, most importantly cancer. Sperm cells are efficient natural microswimmers that can propagate in various liquids, whether elastic or viscous, Newtonian or non-Newtonian (Gaffney et al. 2011). Consequently, the utilization of sperm as motile drug carriers for therapeutic in vivo applications is not too far-fetched. This especially stands to reason for the treatment of diseases in the female reproductive tract, for example, cervical cancer, as this is also the environment in which sperm are naturally adapted to thrive. While the vaginal drug delivery route has been known since ancient times (Hussain \& Ahsan 2005), the idea of utilizing sperm-hybrid micro-bio-robot for fertilization as well as the possibility to use the synthetic attachment to carry anti-cancer drugs have been conceived (Magdanz \& Schmidt 2014). Therapeutics may be attached to sperm cells together with synthetic attachments to guide their motion or incorporated into the sperm head to take advantage of the sperm's abilities to protect molecular cargo and deliver it from cell to cell (Zoabi et al. 2013). Such a target cell might also be the oocyte; for example, to deliver genes or diagnostic tools with the sperm directly in the process of fertilization. However, in this case one needs to be careful to avoid impeding the sperm cells' ability to fertilize and especially in the case of human medicine - in relation to ethical concerns (Barkalina et al. 2016). To the best of our knowledge, the first coherent example of the delivery of synthetic agents in the process of fertilization with sperm cells was conduct with murine sperm and oocytes (Geerts et al. 2014). Lipid vesicles were loaded with green fluorescent protein (GFP)-mRNA and bound to the sperm head and midpiece. Conventional IVF was conducted and the delivery of the fluorescent marker to the oocyte was verified (Fig. 5A).

A sperm-hybrid system with an application in cervical cancer treatment - completely dissociated from fertilization - has been more recently presented by Schmidt and Medina-Sánchez's group (Xu et al. 2018). Here, the therapeutic cargo - the chemotherapeutic doxorubicin (DOX) - was loaded directly into the heads of bovine sperm cells, where it presumably binds to DNA in the cells' nuclei. It was shown that the DOX does not harm a sperm cell or impede its ability to swim, most likely due to the inherent lack of metabolism of the gamete, which makes it invulnerable to the chemotherapeutic. Moreover, the DOX was shown to be delivered to target tumor cells via cell fusion, and the therapeutic effect was verified and compared with DOX in solution (Fig. 5B). Additionally, a synthetic support structure called Tetrapod was attached to individual sperm cells to guide the sperm-hybrid drug carriers magnetically, similar to the sperm-hybrid micro-bio-robot for fertilization discussed above. The distinctive feature of the Tetrapod structure is its ability to release the confined sperm cell in response to a mechanical trigger, that is, by hitting the target tumor spheroid. The four arms of the Tetrapod that prevent the captured sperm cell from swimming through and out of the tubular body of the synthetic microstructure during navigation were designed to bend outward when the sperm-hybrid microcarrier hits an obstacle, that is, the target cells (Fig. 5B and Video 2). This elastic deformation opens sufficient space for the sperm cell to escape from the Tetrapod and penetrate the tumor spheroid ahead to fuse with cancer cells and release its chemotherapeutic cargo. This principle could also be applied to deliver genes to oocytes - like in the previously discussed study - while the Tetrapod enables magnetic guidance. Hereby, in comparison with magnetic nanoparticles that could be attached to the sperm membrane to allow magnetic manipulation, an advantage of the bulky synthetic attachment is that no synthetic material apart from the biomolecular cargo would be brought into the oocyte upon fertilization when the sperm is released. In order to bring this technology closer to the clinic, Schmidt and MedinaSánchez's group have recently demonstrated the transfer of this concept to human sperm (Xu et al. 2019). DOX was successfully loaded into human sperm cells, and the drug was localized predominantly in the sperm nucleus, verified by high-resolution optical microscopy. $3 \mathrm{D}$ cervical cancer and patient-representative 3D ovarian cancer tumors were treated in vitro with those drug-loaded human sperms and showed high cancerkilling efficiency compared with the control samples. Moreover, a new concept of carriers for multiple sperm cells was introduced for the first time to enhance the dosage control of the administrated drug.

\section{Video 2}

Micromotor-assisted drug-loaded sperm cargodelivery. The video (http:// movie-usa.glencoesoftware. com/video/10.1530/REP- 19-0096/video-1) from the online version of the article is available at https://doi. org/10.1530/REP-19-0096.

In a different work focusing on drug delivery detached from fertilization, DOX was also directly incorporated into the sperm heads, although here via DOX-coated iron oxide nanoparticles that attached onto the cell membrane and were endocytosed (Chen et al. 2018). Furthermore, sperm-hybrid microswimmers with different endocytosed quantum dots and fluorescent nanoparticles were presented to show the method's versatility. In this study, the synthetic components served as therapeutic cargo or potentially as biomarkers or mediators for hyperthermia treatment (Fig. 5C). However, the guidance of the sperm-hybrid devices which is necessary for targeted delivery - was solely 
A
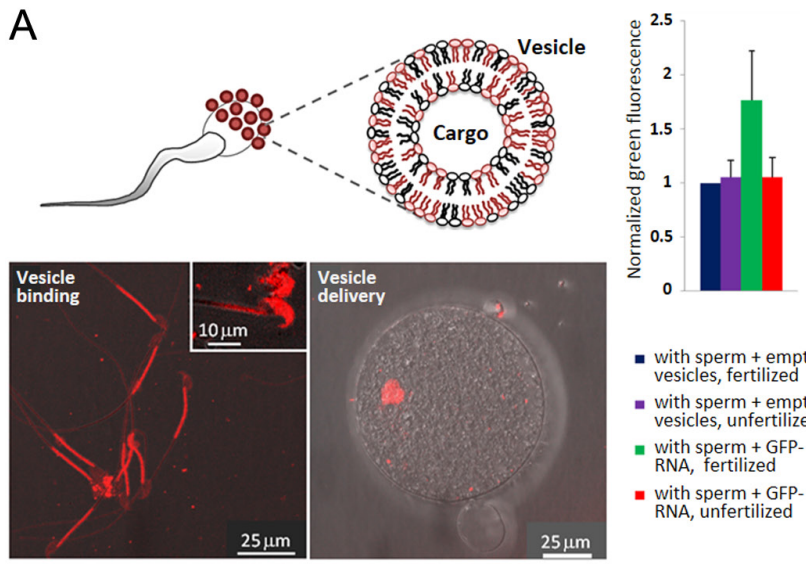
with sperm + empty
vesicles, fertilized

with sperm + empty
vesicles, unfertilized

with sperm + GFP-

RNA, fertilized

- with sperm + GFP-
B

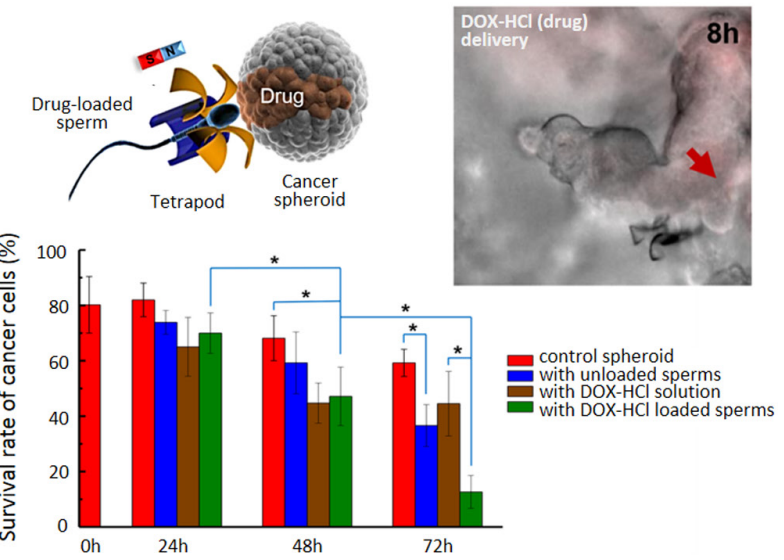

D
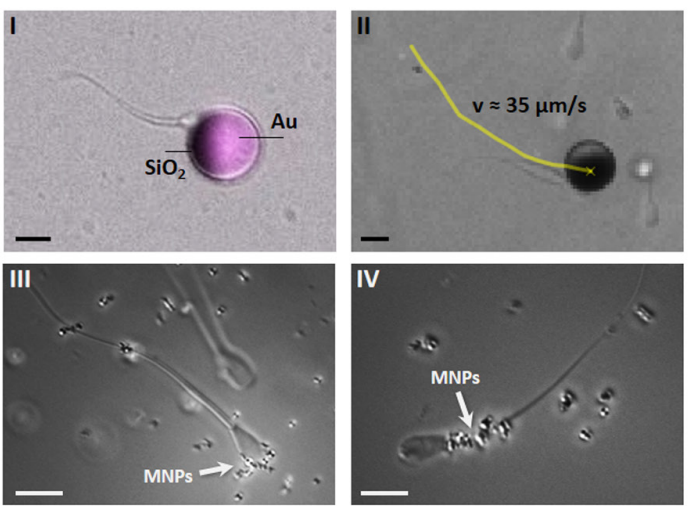

MNPs: magnetic nanoparticles

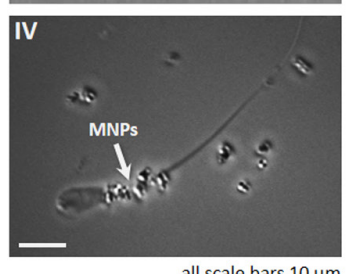

Figure 5 Therapeutic delivery of synthetic cargo by sperm-hybrid micromotors. (A) Functionalization of murine sperm cells with cargo-loaded liposomes and delivery of GFP to murine oocytes (Geerts et al. 2014). (B) Coupling of magneto- and mechanosensitive Tetrapod microstructure to DOX-loaded bovine sperm cell for controlled guidance and release into cancer spheroids and subsequent drug transfer by cell fusion (see also Video 2) (Xu et al. 2018). (C) Loading of different synthetic cargo particles into ascidian sperm cells by endocytosis and targeted delivery to a suitable chemoattractant source (Chen et al. 2018). (D) Attachment of one (I) and two (II) bovine sperm cells to $\mathrm{SiO}_{2} / \mathrm{Au}_{\mathrm{u}}$ Janus particles of $20 \mu \mathrm{m}$ diameter, binding of magnetic nanoparticles to bovine sperm head (III) and midpiece (IV), indicated by arrows.

relinquished to the sperm's chemotaxis ability. Therefore, ascidian sperm was employed. The sea squirt's sperm cells are known to rely much more on chemotaxis than other orientation strategies, in comparison with mammalian sperm, because these marine species' sperm cells have to travel through sea water before they arrive the female and thus cannot so strongly rely on other clues that mammalian sperm would derive from their surroundings in the female reproductive tract. The chemotactic response of the sperm-hybrid microswimmers of the discussed study was confirmed with a suitable chemoattractant extracted from the same species' egg cells (Fig. 5C). In principle, chemotactic targeting is an elegant approach for in vivo drug delivery if the employed microorganism is sensitive to the desired site for treatment. If possible and reliable, such an on-board navigation system is preferable to external control methods like magnetic guidance, because no synthetic mediators are necessary and elaborate visual feedback - which is especially difficult for deep- tissue in vivo applications - might be unnecessary. In the presented study, the tumor-killing efficacy of DOX released from sperm-hybrid carriers was indicated by the reduced viability of targeted human ovarian cancer cells, although tumor- or cell-specific targeting by chemotaxis of the employed ascidian sperm was not shown. Moreover, for sperm from different species that are to be applied for in vivo drug delivery as mentioned in the discussed example, it is necessary to ensure nontoxicity as well as viability of the sperm cells in the human body. For example, sperm from marine species like sea squirts are probably not used to operating at $37^{\circ} \mathrm{C}$ body temperature.

In order to reduce the number of sperm cells necessary for efficient drug delivery, it may be advantageous to significantly increase the cargo-to-sperm ratio. Instead of loading their heads or functionalizing their surface, sperm can be also employed to tow loads that are much heavier than their own body. As has already been shown with several bacteria species (Schwarz et al. 2017), 
one or multiple flagellated microswimmers can carry particles of several tens of micrometers in diameter. In Fig. 5D, this is illustrated by an experiment in our laboratory where one (i) and two (ii) bovine sperm cells attached to silica/gold $\left(\mathrm{SiO}_{2} / \mathrm{Au}\right)$ Janus particles of $20 \mu \mathrm{m}$ diameter. The two sperm cells in Fig. 5D, II were able to push the particle with more than 100 -fold of their weight with a moderate velocity of ca. $35 \mu \mathrm{m} / \mathrm{s}$. Considering assisted reproduction with sperm-hybrid micromotors, a similar approach may be employed to magnetically guide multiple sperm cells, all attached to one single synthetic entity. Such engineered sperm flocks would not only help to raise the fertilization success probability - with more sperm cells at the target site - but also reduce the necessary amount of synthetic material and potentially simplify in vivo tracking and imaging. However, while adsorption to a spherical model particle is simple and illustrative, a more sophisticated design is desirable that allows the robust and reversible coupling and parting of biological and synthetic components, especially for fertilization, but also for drug delivery applications where the synthetic drug container must also first protect and then release its load at the target site. Stimuli-triggered systems like thermoresponsive materials and mechanoresponsive architectures were discussed earlier as examples of such a strategy.

Finally, as opposed to Chen et al.'s work on ascidian sperm cells that were functionalized and permeated with nanoparticles all over their body (Chen et al. 2018), one might only target selective parts of the sperm cell to interfere with the natural organism as little as possible. For example, Medina-Sánchez envisioned the idea of functionalizing only the sperm tail or midpiece with magnetic micro- or nanoparticles by selecting suitable antibodies for covalent binding (Magdanz et al. 2017). The selective functionalization of parts of the sperm tail is especially interesting for improving sperm's swimming performance. In the case of immotile sperm cells, the integration of magnetic particles with the midpiece or tail may be used to actuate the flagellum, as the applied magnetic torque can be translated to an alternating beating pattern at a suitable frequency, similar to the one observed in motile sperm. Moreover, if the particles are aligned with a preferential orientation during the immobilization process, they can be used to guide motile sperm cells magnetically. In both cases, the proposed manipulation mechanism may facilitate the penetration of cumulus cells while limiting the interference of the synthetic components with the sperm head and its DNA content or the control of drug doses by means of multiple sperm transport in the case of drug-carrying sperm.

In general, the functionality of sperm in different environments is decisive for the applicability of spermhybrid micromotors. For fertilization, sperm cells may only need selective assistance in an environment for which they are principally fit, although for drug delivery they need to act as the driving force for tasks that they are not evolutionarily adapted to accomplish. Whereas mammalian sperm cells may be well suited as drug carriers to treat tumors or other diseases in the female reproductive tract (as in Xu et al. 2018), drug delivery applications in other parts of the body require spermhybrid microcarriers to swim in other body fluids, most importantly blood. In principle, this is possible due to the ability of sperm to swim in high-viscosity fluids (Gaffney et al. 2011), although it is necessary to investigate sperm viability in different body fluids as well as non-toxicity to the respective in vivo environment in the future, especially considering sperm from different species.

Besides swimming and cargo transport, the tracking and visualization of sperm-hybrid micromotors are integral for their successful operation, not only for efficient, remote-controlled guidance, but also to supervise sperm behavior and viability. Deep-tissue realtime in vivo imaging of micromotors is a major challenge in the community. Box 2 provides a brief summary of the current state of the art in this field.

Whereas in vivo animal studies with micromotors are already reported toward targeted drug delivery applications (Gao et al. 2015, Servant et al. 2015), the in vivo applicability of sperm-hybrid micromotors for assisted reproduction is yet to be confirmed to justify its impact on the future of reproductive medicine. Regarding individual magnetic micromotors, first studies on realtime tracking below $1 \mathrm{~cm}$ thick phantom tissues have been reported with photoacoustic imaging, opening the

Box 2: In vivo imaging of biomedical micromotors.

Ultrasound sonography is a well-known imaging technology, especially in reproductive medicine. It already offers deep-tissue penetration and images in real time at doses that are not harmful to the body. However, its resolution is poor, at least if one wants to visualize microscopic structures (Ng and Swanevelder 2011). The main problem is a low signal-to-noise ratio due to scattering, which is currently sought out to be improved by employing so-called echogenic contrast agents that may serve as signal amplifiers (Zlitni and Gambhir 2018). Other methods with similar strengths and weaknesses are magnetic resonance imaging (MRI) and magnetic particle imaging (MPI) (Weizenecker et al. 2009). Although large and expensive instruments are needed for both, they may benefit from the advantage that magnetically propelled micromotors may be actuated by the same device and simultaneously act as tracer particles (Martel et al. 2009). This is important as the in vivo imaging setup - just like the micromotor itself - needs to be compliant and feasible in the clinical routine. Fluorescence imaging generally offers much higher resolution at the cost of poor tissue penetration (Wang et al. 2018). Approaches that combine the strengths of several techniques - for example, photoacoustic imaging - may be able to push these limits in the future (Medina-Sánchez and Schmidt 2017). Nonetheless, possible health concerns - such as with radioactive substances in diagnostic nuclear medicine - always need to be considered when a novel technique or combination of techniques is proposed. 
Table 2 Published sperm-hybrid systems in the micromotor community.

\begin{tabular}{|c|c|c|c|c|c|}
\hline Reference & $\begin{array}{l}\text { Sperm } \\
\text { origin }\end{array}$ & Synthetic component & Component size & Intended application & $\begin{array}{l}\text { Purpose of synthetic } \\
\text { component }\end{array}$ \\
\hline Magdanz et al. 2013 & Bovine & $\begin{array}{l}\text { Metal/metal } \\
\text { microtube }\end{array}$ & Tube length $\approx 50 \mu \mathrm{m}$ & $\begin{array}{l}\text { Assisted } \\
\text { reproduction }\end{array}$ & Magnetic guidance \\
\hline Geerts et al. 2014 & Murine & $\begin{array}{l}\text { Protein-loaded lipid } \\
\text { vesicles }\end{array}$ & Vesicle size $\approx 200 \mathrm{~nm}$ & $\begin{array}{l}\text { Intraoocyte gene } \\
\text { delivery }\end{array}$ & $\begin{array}{l}\text { Cargo containers, } \\
\text { fluorescence markers }\end{array}$ \\
\hline Magdanz et al. 2015b & Bovine & $\begin{array}{l}\text { Metal/oxide } \\
\text { microtube }\end{array}$ & Tube length $\approx 208 \mu \mathrm{m}$ & $\begin{array}{l}\text { Assisted } \\
\text { reproduction }\end{array}$ & Magnetic guidance \\
\hline $\begin{array}{l}\text { Medina-Sánchez et al. } \\
2016\end{array}$ & Bovine & $\begin{array}{l}\text { Polymer/metal } \\
\text { microhelix }\end{array}$ & Helix length $\approx 25 \mu \mathrm{m}$ & $\begin{array}{l}\text { Assisted } \\
\text { reproduction }\end{array}$ & $\begin{array}{l}\text { Magnetic propulsion and } \\
\text { release }\end{array}$ \\
\hline Magdanz et al. 2016 & Bovine & $\begin{array}{l}\text { Polymer/metal } \\
\text { microtube }\end{array}$ & Tube length $\approx 50 \mu \mathrm{m}$ & $\begin{array}{l}\text { Assisted } \\
\text { reproduction }\end{array}$ & $\begin{array}{l}\text { Magnetic guidance and } \\
\text { thermoresponsive release }\end{array}$ \\
\hline Xu et al. 2018 & Bovine & $\begin{array}{l}\text { Polymer/metal } \\
\text { Tetrapod tube }\end{array}$ & Tube length $\approx 40 \mu \mathrm{m}$ & $\begin{array}{l}\text { Gynecological drug } \\
\text { delivery }\end{array}$ & $\begin{array}{l}\text { Magnetic guidance and } \\
\text { mechanical release }\end{array}$ \\
\hline Chen et al. 2018 & Ascidian & $\begin{array}{l}\text { Nanoparticles, } \\
\text { quantum dots }\end{array}$ & Particle size $\approx 6-10 \mathrm{~nm}$ & $\begin{array}{l}\text { Chemotactic drug } \\
\text { delivery }\end{array}$ & $\begin{array}{l}\text { Cargo containers, model } \\
\text { drugs }\end{array}$ \\
\hline Xu et al. 2019 & Human & $\begin{array}{l}\text { Polymer/metal } \\
\text { microcap }\end{array}$ & Cap length $\approx 20 \mu \mathrm{m}$ & $\begin{array}{l}\text { Gynecological drug } \\
\text { delivery }\end{array}$ & $\begin{array}{l}\text { Magnetic guidance and } \\
\text { mechanical release }\end{array}$ \\
\hline
\end{tabular}

possibility of applying this technology in small animals for in vivo studies (Aziz et al. 2019). The published works on sperm-hybrid micromotors discussed in this review are listed chronologically and summarized in Table 2.

\section{Investigation of sperm behavior and properties}

The interaction of sperm cell and attached synthetic material is an interesting topic to study in itself: first of all, to study the potential negative effects of materials on sperm viability and function, and second to study sperm behavior in response to external stimuli in general.

Numerous works have studied the swimming behavior of sperm cells, including taking advantage of lab-on-achip approaches that employ microfluidics for sperm sorting and selection (Knowlton et al. 2015, Nosrati et al. 2017, Kashaninejad et al. 2018) or other techniques like tethered robotic micromanipulators (Zhang et al. 2019) and optical tweezers (Ohta et al. 2010) to fixate the motile cells. Sperm-hybrid microdevices offer close interfaces between cell and synthetic material that may serve to study sperm behavior in a more immediate manner. For example, sperm-microtube couplings may provide insights into tail-beating patterns in confined spaces (Magdanz et al. 2015a). The aforementioned potential effect of such synthetic structures on the sperm cells is especially important for fertilization applications, as a resulting embryo has to be free of any undesired influences. For example, it has been shown that silver nanoparticles that were internalized by murine sperm cells had a negative effect on fertilization outcome and embryo development (Yoisungnern et al. 2015). Intriguingly, such results also produced the idea of making use of such adverse effects for novel strategies toward contraception. Although it is unfeasible to render individual sperm cells infertile by synthetic attachments, a sperm-hybrid micromotor could be employed to plant contraceptive substances into the female reproductive tract or to mechanically obstruct the fallopian tube in an innocuous and reversible way.

\section{Conclusions}

New ideas have to be pursued to counter male infertility - mostly semen defects - with ART. The current routine of oocyte aspiration, IVF/ICSI, and embryo transfer is expensive, offers limited suitability and success, and is stressful for the (female) body. Sperm-hybrid micromotors that may operate in vivo are a concept where sperm defects - most importantly motility and orientation problems - can be selectively remedied by synthetic attachments that do not interfere with the sperm's capability to fertilize. This could obviate oocyte aspiration and embryo transfer, as the female gamete could be fertilized in its natural surroundings in the female reproductive tract with the micromotorassisted sperm, providing an update to IUI and as such a potential alternative to ICSI. Proof-of-concept studies to treat oligozoospermia and asthenozoospermia with such methods have been discussed in this review (Magdanz et al. 2013, Medina-Sánchez et al. 2016). Furthermore, other applications of the sperm-hybrid micromotor concept - namely for targeted drug delivery - have been introduced (Chen et al. 2018, Xu et al. 2018). In these works, the sperm cell was dislodged from its fertilization purpose and instead employed as a natural microswimmer to transport synthetic cargo, that is, drugs. In both applications, there are similar key challenges to overcome.

Apart from the technical functionality of the hybrid micromotor, for example, controllable swimming and triggered cargo release, biocompatibility is an important issue. In this context, biocompatibility means nontoxicity of the synthetic materials to the coupled sperm cell as well as the surrounding cells and tissue at the site of application. In the case of assisted reproduction, especially the oocyte and the potential embryo should remain untouched by any harmful synthetic influences as this could impede or alter embryo development in a hardly predictable manner. In the case of drug delivery 
or other applications of sperm-hybrid micromotors in regions of the body where sperm cells are not naturally present, the immune system's response to the cellular intruders also needs to be considered and investigated. Moreover, the use of sperm from different species for such applications might be possible and favorable, although it needs to be handled with care. Most of the cited studies in this review worked with animal sperm for reasons of availability and legal regulations, although it may be the case that different sperm cells have strengths toward specific applications - for example, tumorselective chemotaxis (e.g. aerotaxis, pH-taxis) - that are currently unknown.

In order to guide a sperm-hybrid micromotor to a specific target in vivo - whether for fertilization or another purpose - some means of visualization is needed, either for manual steering by the external operator, or for closed-loop robotic tracking. Thus far, it has been possible to visualize large swarms of micromotors in mice - for example, magnetic helices (Servant et al. 2015) - similar to those that were used for the artificial propulsion of immotile sperm cells discussed in this review. However, the tracking and controlled navigation of individual sperm-hybrid microdevices and targeted delivery to the oocyte inside the fallopian tube of a human patient requires much higher resolution and deep-tissue penetration than what is currently possible with established live-imaging techniques like ultrasound or MRI. Nonetheless, novel techniques and combinations of techniques are being developed that may soon be able to clear this hurdle. However, apart from technical challenges, biocompatibility, medical risks and compliance with clinical standards of such imaging techniques also need to be considered. In general, more studies with micromotors for in vivo assisted reproduction or other biomedical applications need to be conducted in in vivo setups, that is, animal studies, to investigate the functionality, biocompatibility, and controllability of such devices under realistic conditions, instead of limited in vitro experiments. Subsequently, the application of sperm-hybrid micromotors and other biomedical microdevices to locally treat different medical conditions inside the body may soon be able to prove its potential to offer medicine a new drive in the twenty-first century.

\section{Declaration of interest}

The authors declare that there is no conflict of interest that could be perceived as prejudicing the impartiality of this review.

\section{Funding}

This research did not receive any specific grant from any funding agency in the public, commercial or not-for-profit sector.

\section{Acknowledgments}

The authors thank the priority program of the German Research Foundation SPP 1726 'Microswimmers - From Single Particle Motion to Collective Behavior' for financial support. O G S acknowledges financial support by the Leibniz Program of the German Research Foundation. The authors also thank $\mathrm{F}$ Hebenstreit for the technical support on the sperm functionalization using different particle sizes, and $\mathrm{H} \mathrm{Xu}, \mathrm{F}$ Striggow, A Aziz and F Hebenstreit for the fruitful discussions.

\section{References}

Alvarez L 2017 The tailored sperm cell. Journal of Plant Research 130 455464. (https://doi.org/10.1007/s10265-017-0936-2)

Aziz A, Medina-Sánchez M, Claussen J \& Schmidt OG 2019 Real-Time Optoacoustic Tracking of Single Moving Micro-objects in Deep Phantom and Ex Vivo Tissues. Nano Letters 19 6612-6620.

Bahat A \& Eisenbach M 2006 Sperm thermotaxis. Molecular and Cellular Endocrinology 252 115-119. (https://doi.org/10.1016/j. mce.2006.03.027)

Barkalina N, Jones C \& Coward K 2016 Nanomedicine and mammalian sperm: lessons from the porcine model. Theriogenology 85 74-82. (https://doi.org/10.1016/j.theriogenology.2015.05.025)

Bente K, Codutti A, Bachmann F \& Faivre D 2018 Biohybrid and bioinspired magnetic microswimmers. Small 14 e1704374. (https://doi. org/10.1002/smll.201704374)

Bianchi E, Doe B, Goulding D \& Wright GJ 2014 Juno is the egg Izumo receptor and is essential for mammalian fertilization. Nature $\mathbf{5 0 8}$ 483487. (https://doi.org/10.1038/nature13203)

Brannigan RE \& Lipshultz LI 2008 Sperm transport and capacitation. The Global Library of Women's Medicine ISSN: 1756-2228. (https://doi. org/10.3843/GLOWM.10316)

Chang H, Kim BJ, Kim YS, Suarez SS \& Wu M 2013 Different migration patterns of sea urchin and mouse sperm revealed by a microfluidic chemotaxis device. PLOS ONE 8 e60587. (https://doi.org/10.1371/ journal.pone.0060587)

Chen C, Chang X, Angsantikul P, Li J, Esteban-Fernández de Ávila B, Karshalev E, Liu W, Mou F, He S, Castillo R, et al. 2018 Chemotactic guidance of synthetic organic/inorganic payloads functionalized sperm micromotors. Advanced Biosystems 2 1-7. (https://doi.org/10.1002/ adbi.201700160)

Coy P, García-Vázquez FA, Visconti PE \& Avilés M 2012 Roles of the oviduct in mammalian fertilization. Reproduction 144 649-660. (https:// doi.org/10.1530/REP-12-0279)

Cummins JM \& Woodall PF 1985 On mammalian sperm dimensions. Journal of Reproduction and Fertility 75 153-175. (https://doi. org/10.1530/jrf.0.0750153)

Denissenko P, Kantsler V, Smith DJ \& Kirkman-Brown J 2012 Human spermatozoa migration in microchannels reveals boundary-following navigation. PNAS 109 8007-8010. (https://doi.org/10.1073/ pnas.1202934109)

Dreyfus R, Baudry J, Roper ML, Fermigier M, Stone HA \& Bibette J 2005 Microscopic artificial swimmers. Nature 437 862-865. (https://doi. org/10.1038/nature04090)

Fauci LJ \& McDonald A 1995 Sperm motility in the presence of boundaries. Bulletin of Mathematical Biology 57 679-699. (https://doi.org/ 10.1007/ BF02461846)

Friedrich BM \& Jülicher F 2007 Chemotaxis of sperm cells. PNAS 104 13256-13261. (https://doi.org/10.1073/pnas.0703530104)

Gaffney EA, Gadêlha H, Smith DJ, Blake JR \& Kirkman-Brown JC 2011 Mammalian sperm motility: observation and theory. Annual Review of Fluid Mechanics 43 501-528. (https://doi.org/10.1146/annurevfluid-121108-145442)

Gao W, Dong R, Thamphiwatana S, Li J, Gao W, Zhang L \& Wang J 2015 Artificial micromotors in the mouse's stomach : a step toward in vivo use of synthetic motors. ACS Nano 9 117-123. (https://doi.org/10.1021/ nn507097k)

Geerts N, McGrath J, Stronk JN, Vanderlick TK \& Huszar G 2014 Spermatozoa as a transport system of large unilamellar lipid vesicles into 
the oocyte. Reproductive Biomedicine Online 28 451-461. (https://doi. org/10.1016/j.rbmo.2013.11.009)

Ghosh A \& Fischer P 2009 Controlled propulsion of artificial magnetic nanostructured propellers. Nano Letters 9 2243-2245. (https://doi. org/10.1021/nl900186w)

Giustini AJ, Petryk AA, Cassim SM, Tate JA, Baker I \& Hoopes PJ 2010 Magnetic nanoparticle hyperthermia in cancer treatment. NANO Life $\mathbf{1}$ 17-32. (https://doi.org/10.1142/S1793984410000067)

Harvey C 1960 The speed of human spermatozoa and the effect on it of various diluents, with some preliminary observations on clinical material. Journal of Reproduction and Fertility 1 84-95. (https://doi. org/10.1530/jrf.0.0010084)

Hussain A \& Ahsan F 2005 The vagina as a route for systemic drug delivery. Journal of Controlled Release 103 301-313. (https://doi.org/10.1016/j. jconrel.2004.11.034)

Kashaninejad N Shiddiky MJA and Nguyen N-T 2018 Advances in microfluidics-based assisted reproductive technology: from sperm sorter to reproductive system-on-a-chip. Advanced Biosystems 2 1-21. (https:// doi.org/10.1002/adbi.201700197)

Kaupp UB \& Strünker T 2017 Signaling in sperm: more different than similar. Trends in Cell Biology 27 101-109. (https://doi.org/10.1016/j. tcb.2016.10.002)

Khalil ISM, Dijkslag HC, Abelmann L \& Misra S 2014 MagnetoSperm: a microrobot that navigates using weak magnetic fields. Applied Physics Letters 104 1-4. (https://doi.org/10.1063/1.4880035)

Knowlton SM, Sadasivam M \& Tasoglu S 2015 Microfluidics for sperm research. Trends in Biotechnology 33 221-229. (https://doi. org/10.1016/j.tibtech.2015.01.005)

Kromer JA, Märcker S, Lange S, Baier C \& Friedrich BM 2018 Decision making improves sperm chemotaxis in the presence of noise. PLOS Computational Biology 14 e1006109. (https;//doi.org/10.1371/journal. pcbi.1006109)

Lauga E \& Powers TR 2009 The hydrodynamics of swimming microorganisms. Reports on Progress in Physics 72 1-36. (https://doi. org/10.1088/0034-4885/72/9/096601)

Magdanz V \& Schmidt OG 2014 Spermbots: potential impact for drug delivery and assisted reproductive technologies. Expert Opinion on Drug Delivery 11 1125-1129. (https://doi.org/10.1517/17425247.2014.9245 02)

Magdanz V, Sanchez S \& Schmidt OG 2012 Method for the conrolled movement of motile cells in liquid or gaseous media. Patent No. DE102012212427A1 (Germany), US9883889B2 (United States).

Magdanz V, Sanchez S \& Schmidt OG 2013 Development of a spermflagella driven micro-bio-robot. Advanced Materials 25 6581-6588. (https://doi.org/10.1002/adma.201302544)

Magdanz V, Koch B, Sanchez S \& Schmidt OG 2015a Sperm dynamics in tubular confinement. Small 11 781-785. (https://doi.org/10.1002/ smll.201401881)

Magdanz V, Medina-Sánchez M, Chen Y, Guix M \& Schmidt OG 2015b How to improve spermbot performance. Advanced Functional Materials 25 2763-2770. (https://doi.org/10.1002/adfm.201500015)

Magdanz V, Guix M, Hebenstreit F \& Schmidt OG 2016 Dynamic polymeric microtubes for the remote-controlled capture, guidance, and release of sperm cells. Advanced Materials 28 4084-4089. (https://doi. org/10.1002/adma.201505487)

Magdanz V, Medina-Sánchez M, Schwarz L, Xu H, Elgeti J \& Schmidt OG 2017 Spermatozoa as functional components of robotic microswimmers. Advanced Materials 29 1-18. (https://doi.org/10.1002/adma.201606301)

Martel S, Felfoul O, Mathieu JB, Chanu A, Tamaz S, Mohammadi M, Mankiewicz M \& Tabatabaei N 2009 MRI-based medical nanorobotic platform for the control of magnetic nanoparticles and flagellated bacteria for target interventions in human capillaries. International Journal of Robotics Research 28 1169-1182. (https://doi. org/10.1177/0278364908104855)

Medina-Sánchez M \& Schmidt OG 2017 Medical microbots need better imaging and control. Nature 545 406-408. (https://doi. org/10.1038/545406a)

Medina-Sánchez M, Schwarz L, Meyer AK, Hebenstreit F \& Schmidt OG 2016 Cellular cargo delivery: toward assisted fertilization by spermcarrying micromotors. Nano Letters 16 555-561. (https://doi. org/10.1021/acs.nanolett.5b04221)
Medina-Sánchez M, Magdanz V, Schwarz L, Xu H \& Schmidt OG 2017 Spermbots: concept and applications. In Lecture Notes in Computer Science. Eds. M Mangan, M Cutkosky, A Mura, PFMJ Verschure, T Prescott \& N Lepora. Cham: Springer 579-588. (https://doi.org/10.1007/978-3319-63537-8_53)

Medina-Sánchez M, Magdanz V, Guix M, Fomin VM \& Schmidt OG 2018 Swimming microrobots: soft, reconfigurable, and smart. Advanced Functional Materials 28 1-27. (https://doi.org/10.1002/adfm.201707228)

Mei Y, Huang G, Solovev AA, Ureña EB, Mönch I, Ding F, Reindl T, Fu RKY, Chu PK \& Schmidt OG 2008 Versatile approach for integrative and functionalized tubes by strain engineering of nanomembranes on polymers. Advanced Materials 20 4085-4090. (https://doi.org/10.1002/ adma.200801589)

Miki K \& Clapham DE 2013 Rheotaxis guides mammalian sperm. Current Biology 23 443-452. (https://doi.org/10.1016/j.cub.2013.02.007)

Moore H, Dvorakova K, Jenkins N \& Breed W 2002 Exceptional sperm cooperation in the wood mouse. Nature 418 174-177. (https://doi. org/10.1038/nature00832)

Ng A \& Swanevelder J 2011 Resolution in ultrasound imaging. Continuing Education in Anaesthesia Critical Care \& Pain 11 186-192. (https://doi. org/10.1093/bjaceaccp/mkr030)

Nosrati R, Graham PJ, Zhang B, Riordon J, Lagunov A, Hannam TG, Escobedo C, Jarvi K \& Sinton D 2017 Microfluidics for sperm analysis and selection. Nature Reviews: Urology 14 707-730. (https://doi. org/10.1038/nrurol.2017.175)

Ohta AT, Garcia M, Valley JK, Banie L, Hsu HY, Jamshidi A, Neale SL, Lue T \& Wu MC 2010 Motile and non-motile sperm diagnostic manipulation using optoelectronic tweezers. Lab on a Chip 10 3213-3217. (https:// doi.org/10.1039/c0lc00072h)

Purcell EM 1977 Life at low Reynolds number. American Journal of Physics 45 3-11. (https://doi.org/10.1119/1.10903)

Rode S, Elgeti J \& Gompper G 2018 Sperm motility in modulated microchannels. New Journal of Physics 21 1-16. (https://doi. org/10.1088/1367-2630/aaf544)

Saggiorato G, Alvarez L, Jikeli JF, Kaupp UB, Gompper G \& Elgeti J 2017 Human sperm steer with second harmonics of the flagellar beat. Nature Communications 8 1415. (https://doi.org/10.1038/s41467-017-01462-y)

Schmidt OG 2014 Method for Mobilizing Immobilized Cells. Patent No. DE102014201760A1 (Germany), US20170166882A1 (United States).

Schwarz L, Medina-Sánchez M \& Schmidt OG 2017 Hybrid biomicromotors. Applied Physics Reviews 4 031301. (https://doi.org/10.1063/1.4993441)

Servant A, Qiu F, Mazza M, Kostarelos K \& Nelson BJ 2015 Controlled in vivo swimming of a swarm of bacteria-like microrobotic flagella. Advanced Materials 27 2981-2988. (https://doi.org/10.1002/ adma.201404444)

Snook RR 2005 Sperm in competition: not playing by the numbers. Trends in Ecology and Evolution 20 46-53. (https://doi.org/10.1016/j. tree.2004.10.011)

Solovev AA, Mei Y, Bermúdez Ureña E, Huang G \& Schmidt OG 2009 Catalytic microtubular jet engines self-propelled by accumulated gas bubbles. Small 5 1688-1692. (https://doi.org/10.1002/smll.200900021)

Suarez SS 2016 Mammalian sperm interactions with the female reproductive tract. Cell and Tissue Research 363 185-194. (https://doi. org/10.1007/s00441-015-2244-2)

Suarez SS \& Pacey AA 2006 Sperm transport in the female reproductive tract. Human Reproduction Update 12 23-37. (https://doi.org/10.1093/ humupd/dmi047)

Wang B, Zhang Y \& Zhang L 2018 Recent progress on micro- and nanorobots: towards in vivo tracking and localization. Quantitative Imaging in Medicine and Surgery 8 461-479. (https://doi.org/10.21037/ qims.2018.06.07)

Weizenecker J, Gleich B, Rahmer J, Dahnke H \& Borgert J 2009 Threedimensional real-time in vivo magnetic particle imaging. Physics in Medicine and Biology 54 L1-L10. (https://doi.org/10.1088/00319155/54/5/L01)

Xu H, Medina-Sánchez M, Magdanz V, Schwarz L, Hebenstreit F \& Schmidt OG 2018 Sperm-hybrid micromotor for targeted drug delivery. ACS Nano 12 327-337. (https://doi.org/10.1021/acsnano.7b06398)

Xu H, Medina-Sánchez M, Brison DR, Edmondson RJ, Taylor SS, Nelson L, Zeng K, Bagley S, Ribeiro C, Restrepo LP, et al. 2019 Human spermbots for cancer-relevant drug delivery. Arxiv 190412684. 
Yang Y, Elgeti J \& Gompper G 2008 Cooperation of sperm in two dimensions: synchronization, attraction, and aggregation through hydrodynamic interactions. Physical Review: E, Statistical, Nonlinear, and Soft Matter Physics 78 061903. (https://doi.org/10.1103/PhysRevE.78.061903)

Yoisungnern T, Choi YJ, Han JW, Kang MH, Das J, Gurunathan S, Kwon DN, Cho SG, Park C, Chang WK, et al. 2015 Internalization of silver nanoparticles into mouse spermatozoa results in poor fertilization and compromised embryo development. Scientific Reports 511170. (https://doi.org/10.1038/srep11170)

Zhang L, Abbott JJ, Dong L, Kratochvil BE, Bell D \& Nelson BJ 2009 Artificial bacterial flagella: fabrication and magnetic control. Applied Physics Letters 94 1-3 (https://doi.org/10.1063/1.3079655)

Zhang Z, Dai C, Huang J, Wang X, Liu J, Ru C, Pu H, Xie S, Zhang J, Moskovtsev S, et al. 2019 Robotic immobilization of motile sperm for clinical intracytoplasmic sperm injection. IEEE Transactions on Bio-Medical Engineering 66 444-452. (https://doi.org/10.1109/ TBME.2018.2848972)
Zlitni A \& Gambhir SS 2018 Molecular imaging agents for ultrasound. Current Opinion in Chemical Biology 45 113-120. (https://doi. org/10.1016/j.cbpa.2018.03.017)

Zoabi N, Golani-Armon A, Zinger A, Reshef M, Yaari Z, Vardi-Oknin D, Shatsberg Z, Shomar A, Shainsky-Roitman J \& Schroeder A 2013 The evolution of tumor-targeted drug delivery: from the EPR effect to nanoswimmers. Israel Journal of Chemistry 53 719-727. (https://doi. org/10.1002/ijch.201300061)

Received 1 March 2019

First decision 24 May 2019

Revised manuscript received 4 September 2019

Accepted 30 September 2019 